\title{
FREQUÊNCIA DE SÍNDROME METABÓLICA EM ESCOLARES
}

\author{
Ana Cláudia Kapp Titski \\ Universidade Federal do Paraná, Curitiba, Paraná, Brasil \\ Deise Cristiane Moser \\ Universidade Federal do Paraná, Curitiba, Paraná, Brasil \\ Fabricio Cieslak \\ Universidade Federal do Paraná, Curitiba, Paraná, Brasil \\ Luis Paulo Gomes Mascarenhas \\ Universidade Federal do Paraná, Curitiba, Paraná, Brasil \\ Manoel João Coelho e Silva \\ Universidade Federal do Paraná, Curitiba, Paraná, Brasil \\ Neiva Leite \\ Universidade Federal do Paraná, Curitiba, Paraná, Brasil
}

\section{Resumo}

Objetivo: Comparar a frequência de síndrome metabólica (SM) em crianças e adolescentes com e sem excesso de peso de uma escola de Curitiba. Métodos: Participaram 182 indivíduos, entre 10 e 16 anos de idade. A SM foi classificada pela presença de 3 ou mais dos critérios do Adult Treatment Panel III (ATPIII), adaptado por Leite et al (2009). Foi utilizado o teste T Student e Qui-Quadrado $(\mathrm{p}<0,05)$. Resultados: A SM ocorreu em 13,7\% com proporções semelhantes entre os gêneros $\left(\chi^{2}=0,12 ; \mathrm{p}=0,913\right)$. Foi observada maior freqüência de SM nos escolares com excesso de peso em relação aos com peso adequado $\left(\chi^{2}=36,019 ; \mathrm{p}=0,00\right)$. Os componentes mais alterados foram: HDL-c e circunferência abdominal. Conclusão: $\mathrm{Ob}$ servou-se elevada proporção de SM em crianças e adolescentes com excesso de peso.

Palavras-chave: Criança. Adolescente. Obesidade. Circunferência Abdominal. Resistência à Insulina.

\section{Introdução}

A síndrome metabólica é caracterizada pela presença de vários fatores de risco cardiovasculares que ocorrem simultaneamente ${ }^{(1)}$. As 
mudanças no estilo de vida contemporâneo provocaram aumento na frequência de síndrome metabólica, tanto em crianças quanto em adultos ${ }^{(2)}$, devido ao crescimento nas taxas de sedentarismo e excesso de peso $^{(3)}$. $\mathrm{O}$ aumento do tecido adiposo visceral é o componente de maior valor preditivo, pois é o responsável pela presença do processo inflamatório e de resistência à insulina ${ }^{(4 ; 5)}$.

A prevalência de síndrome metabólica é variável, em função dos diferentes critérios diagnósticos utilizados. Na população pediátrica, as frequências de síndrome metabólica variam de $4,2 \%{ }^{(7)}$ a $9,2 \%{ }^{(8)}$, com aumento na prevalência quando se consideram crianças e adolescentes obesos para $17,3 \%{ }^{(9)}$ e $35,5 \%{ }^{(7)}$. Alguns estudos destacam que os componentes mais frequentes no diagnóstico de síndrome metabólica são a aumentada circunferência abdominal $(88,1 \%)$ e pressão arterial $(47,5 \%)^{(10)}$, seguidos de maior concentração de triacilglicerol $(23,4 \%)$ e de baixo HDL-colesterol $(23,3 \%)^{(8)}$.

Considerando-se que o excesso de peso tem afetado um terço das crianças e adolescentes entre 10 e 16 anos de idade da rede municipal de Curitiba $-\mathrm{PR}^{(11)}$, a avaliação de escolares possibilita o diagnóstico e intervenção precoces nas comorbidades evitando a evolução desfavorável e o desenvolvimento de doença cardiovascular com maior morbidade e mortalidade na vida adulta ${ }^{(12)}$.

Portanto, o objetivo do estudo foi avaliar a frequência dos componentes da síndrome metabólica nos escolares de 10 a 16 anos da rede municipal de Curitiba - PR, relacionando-a com o gênero e perfil do IMC.

\section{Método}

Estudo descritivo de corte transversal, realizado em escolares de $5^{\mathrm{a}}$ a $8^{\mathrm{a}}$ série de uma escola municipal de Curitiba, Paraná. A amostra foi selecionada por conveniência, avaliando-se os escolares que aceitaram participar da pesquisa e que obtiveram autorização dos pais ou responsáveis, mediante a apresentação do Termo de Consentimento Livre e Esclarecido, de acordo com a Resolução 196/96 do Conselho Nacional de Saúde.

Aprovado pelo Comitê de Ética em Pesquisa do Setor de Ciências da Saúde da Universidade Federal do Paraná, sob o número 403.083.07.07, e realização autorizada pela Secretaria Municipal de Educação de Curitiba - PR.

A amostra calculada foi de 161 alunos (EpiInfo versão 3.5.1), com base no número de escolares regularmente matriculados na escola avaliada $(\mathrm{n}=1300)$, prevalência de síndrome metabólica igual a $45 \%, 95 \%$ de confiança e erro amostral de 5\%. Inicialmente, foram avaliados 205 alunos. Destes, 23 não fizeram parte deste estudo porque não se adequaram aos critérios de inclusão: idade entre $10 \mathrm{e}$ 16 anos $(n=1)$ e realização de todas as avaliações $(n=22)$. A amostra final do estudo foi composta por 182 crianças e adolescentes, sendo 71 meninos e 111 meninas. 
As técnicas utilizadas para obtenção das medidas antropométricas foram realizadas conforme Lohman et al. ${ }^{(13)}$. A estatura foi mensurada em centímetros $(\mathrm{cm})$, ao final de uma inspiração máxima, utilizando-se estadiômetro de parede. $\mathrm{O}$ indivíduo permanecia em posição ortostática, com os pés descalços e unidos, com as superfícies posteriores do calcanhar, cinturas pélvica e escapular e região occipital em contato com a parede, e com a cabeça no plano horizontal de Frankfort.

A massa corporal foi aferida em quilogramas $(\mathrm{kg})$, em balança do tipo plataforma. $\mathrm{O}$ indivíduo mantinha-se descalço, posicionado em pé no centro da plataforma, com os braços ao longo do corpo e utilizando somente o uniforme, sem casaco ou objetos nos bolsos. O peso do uniforme não foi descontado do valor da massa corporal do indivíduo.

Em seguida, calculou-se o índice de massa corporal $(\mathrm{kg} / \mathrm{m} 2)$ pela divisão da massa corporal pela estatura ao quadrado. Os indivíduos foram classificados segundo os critérios definidos pelo Center for Disease Control and Prevention $(\mathrm{CDC})^{(14)}$, para obesidade (percentil acima de $95^{\circ}$ ) conforme sexo, idade e etnia. Além disso, o IMC foi convertido para IMC-escore Z, utilizando-se o IMC de cada indivíduo subtraído do valor correspondente ao $50^{\circ}$ percentil do IMC, dividido pelo desvio-padrão populacional, conforme valores disponibilizados pelo CDC, para cada faixa etária e sexo.

A medida da circunferência abdominal foi realizada com a utilização de uma fita antropométrica flexível e inextensível. O instrumento foi aplicado sobre a pele e na altura das cristas ilíacas, paralelamente ao solo, com o sujeito em pé, o abdome relaxado, os braços ao longo do corpo e os pés unidos. A classificação da circunferência abdominal foi baseada nos critérios propostos por Fernandez et $a l .{ }^{15}$ para todas as etnias, considerando-se os valores acima ou iguais ao $75^{\circ}$ percentil como limítrofes ou aumentados, de acordo com a idade e o gênero.

A mensuração das pressões arteriais sistólica (PAs) e diastólica (PAd) foram realizados com o indivíduo sentado, em repouso. Foram obtidas três medidas com intervalo de dois minutos entre elas, e considerado o valor médio entre as duas últimas medidas para a análise dos níveis pressóricos. A pressão arterial foi considerada elevada quando atingia valores acima ou iguais ao $90^{\circ}$ percentil ou a $120 / 80 \mathrm{mmHg}$, de acordo com as tabelas específicas em percentis para crianças e adolescentes, por idade, gênero e percentil de estatura ${ }^{(16)}$.

A avaliação puberal foi realizada conforme a auto-avaliação de Tanner ${ }^{(17)}$. As meninas e os meninos foram avaliados quanto à pilificação pubiana (P1-P5). Esta avaliação foi realizada individualmente e de forma indireta por pesquisadores do mesmo gênero da criança ou do adolescente.

Os exames laboratoriais foram realizados no período da manhã, após um jejum de 12 horas. Foram coletados $8 \mathrm{ml}$ de sangue por punção venosa para a dosagem do perfil lipídico (LDL-c, HDL-c e triacilglicerol). As concentrações de glicose, lipoproteína de alta densidade -colesterol (HDL-C) e triacilglicerol (TG) foram realizadas em laboratório de Curitiba - PR. 
Para caracterizar a síndrome metabólica, utilizou-se os critérios do Nathional Cholesterol Education Program's Adult Treatment Panel III (NCEP/ATPIII), adaptado por Leite et $a l .{ }^{(18)}$, cujos pontos de corte utilizados correspondem à idade e gênero considerando a presença de três ou mais dos seguintes fatores de risco cardiovasculares: HDL-c $<45 \mathrm{mg} / \mathrm{dL}$; triacilglicerol $\geq 100 \mathrm{mg} / \mathrm{dL}$; circunferência abdominal $\geq$ percentil 75 ; concentração de glicose $\geq 100 \mathrm{mg} / \mathrm{dL}$ e pressão arterial sistólica e/ou diastólica $\geq$ percentil 90 .

$\mathrm{Na}$ análise estatística, utilizou-se o SPSS 13.0 e o Minitab 15.0. Inicialmente foi determinada a normalidade dos dados através do teste de Ryan-Joiner, as variáveis de estatura, PAS e glicemia de jejum se manifestaram dentro da normalidade. Foi verificado os outliers por meio de Boxplots e a única variável que apresentou esses dados discrepantes foi o triacilglicerol.

Para comparar os gêneros em relação às variáveis idade decimal, massa corporal, estatura, índice de massa corporal, circunferência abdominal, pressão arterial sistólica e diastólica, glicemia de jejum, HDL-c e triacilglicerol utilizaramse os testes $t$ de Student independente e U de Mann Withney. O Teste Qui-Quadrado foi utilizado para investigar a associação da síndrome metabólica com os gêneros e o perfil do índice de massa corporal. Considerou-se nível de significância de $\mathrm{p}<0,05$.

\section{Resultados}

Neste estudo participaram 182 escolares, dos quais $60,9 \%$ eram do gênero feminino e a média de idade foi $12,4 \pm 1,48$. Na comparação entre os gêneros, a glicemia em jejum foi maior nos meninos em relação às meninas $(p<0,05)$. As demais variáveis foram semelhantes entre os gêneros (Tabela 1). 
Tabela 1: Características da amostra, conforme os Gêneros

\begin{tabular}{|c|c|c|c|c|}
\hline Variáveis & $\begin{array}{c}\text { Meninos } \\
(\mathrm{n}=71)\end{array}$ & $\begin{array}{l}\text { Meninas } \\
(\mathrm{n}=111) \\
\end{array}$ & $\begin{array}{c}\text { Total } \\
(\mathrm{n}=182) \\
\end{array}$ & $\boldsymbol{P}$ \\
\hline Idade decimal (anos) & $12,54 \pm 1,5$ & $12,39 \pm 1,48$ & $12,4 \pm 1,48$ & 0,521 \\
\hline Massa corporal (kg) & $49,1 \pm 13,8$ & $48,2 \pm 12,53$ & $48,56 \pm 13,01$ & 0,646 \\
\hline Estatura $(\mathrm{m})$ & $1,54 \pm 0,12$ & $1,52 \pm 0,09$ & $1,53 \pm 0,1$ & 0,283 \\
\hline Índice de massa corporal $\left(\mathrm{kg} / \mathrm{m}^{2}\right)$ & $20,32 \pm 3,64$ & $20,5 \pm 4,17$ & $20,46 \pm 3,96$ & 0,703 \\
\hline Circunferência abdominal $(\mathrm{cm})$ & $70,9 \pm 10,12$ & $71,8 \pm 10,28$ & $71,4 \pm 10,2$ & 0,587 \\
\hline $\begin{array}{l}\text { Pressão } \\
(\mathrm{mmHg})\end{array} \quad$ arterial $\quad$ sistólica & $98 \pm 11,3$ & $97 \pm 11$ & $97 \pm 11,4$ & 0,422 \\
\hline $\begin{array}{l}\text { Pressão } \\
(\mathrm{mmHg})\end{array}$ arterial diastólica & $62 \pm 12,1$ & $61 \pm 11,2$ & $61 \pm 11,58$ & 0,641 \\
\hline Glicemia de jejum (mg/dL) & $87,3 \pm 7,8$ & $84,6 \pm 7,4$ & $85,6 \pm 7,7$ & $0,023^{*}$ \\
\hline HDL-colesterol (mg/dL) & $43,6 \pm 10,4$ & $43,2 \pm 10,7$ & $43,4 \pm 10,6$ & 0,822 \\
\hline Triacilglicerol (mg/dL) & $78,4 \pm 46$ & $79,34 \pm 32,14$ & $78,9 \pm 38,03$ & 0,873 \\
\hline
\end{tabular}

NOTA: Valores expressos em médias \pm desvio-padrão; (*) significância estatística ao nível de $p<0,05$.

A frequência de síndrome metabólica e de seus componentes foi, primeiramente, analisada em toda a amostra. Os resultados mostraram que a síndrome metabólica esteve presente em $13,7 \%$ das crianças e adolescentes deste estudo, e que os componentes mais freqüentemente alterados foram o HDL-colesterol (63,7\%), a circunferência abdominal (33,5\%) e o triacilglicerol (20,3\%). Quando os gêneros foram comparados, não se verificaram diferenças significativas ( $p>0,05)$ nos percentuais de síndrome metabólica $\left(\chi^{2}=0,12 ; p=0,913\right)$, e das alterações da circunferência abdominal $\left(\chi^{2}=0,066 ; p=0,798\right)$, pressão arterial $\left(\chi^{2}=0,271\right.$; $\mathrm{p}=0,603)$, glicemia de jejum $\left(\chi^{2}=0,952 ; \mathrm{p}=0,329\right)$, HDL-colesterol $\left(\chi^{2}=1,057\right.$; $\mathrm{p}=0,304)$ e triacilglicerol $\left(\chi^{2}=0,046 ; \mathrm{p}=0,831\right)($ GRÁFICO 1$)$. 
Gráfico 1: Distribuição da síndrome metabólica e de seus componentes, conforme o gênero

Analisando-se o perfil do IMC, verificou-se $62,1 \%$ de eutrofia, quase um terço de sobrepeso $(28,6 \%)$ e $9,3 \%$ de obesidade dentre as crianças e adolescentes estudados. Na comparação dos gêneros, houve maior frequência de sobrepeso entre os meninos e maior percentual de obesidade entre as meninas, embora sem diferença significativa $\left(\chi^{2}=4,705 ; \mathrm{p}=0,095\right)$ (GRÁFICO 2).

Gráfico 2: Distribuição do índice de massa corporal, conforme o gênero

Ao categorizar a amostra pelo perfil do IMC, foi possível verificar que os escolares com excesso de peso apresentaram maiores percentuais de síndrome metabólica $\left(\chi^{2}=36,019 ; \mathrm{p}=0,000\right)$, obesidade abdominal $\left(\chi^{2}=106,423 ; \mathrm{p}=0,000\right)$, pressão arterial elevada $\left(\chi^{2}=9,990 ; \mathrm{p}=0,002\right)$, HDL-colesterol baixo $\left(\chi^{2}=8,220\right.$; $\mathrm{p}=0,004)$ e hipertrigliceridemia $\left(\chi^{2}=9,160 ; \mathrm{p}=0,002\right)$, quando comparados aos eu 
tróficos. A glicemia de jejum elevada foi a única variável que se apresentou semelhante entre os grupos $\left(\chi^{2}=0,701 ; p=0,403\right)$ (GRÁFICO 3).

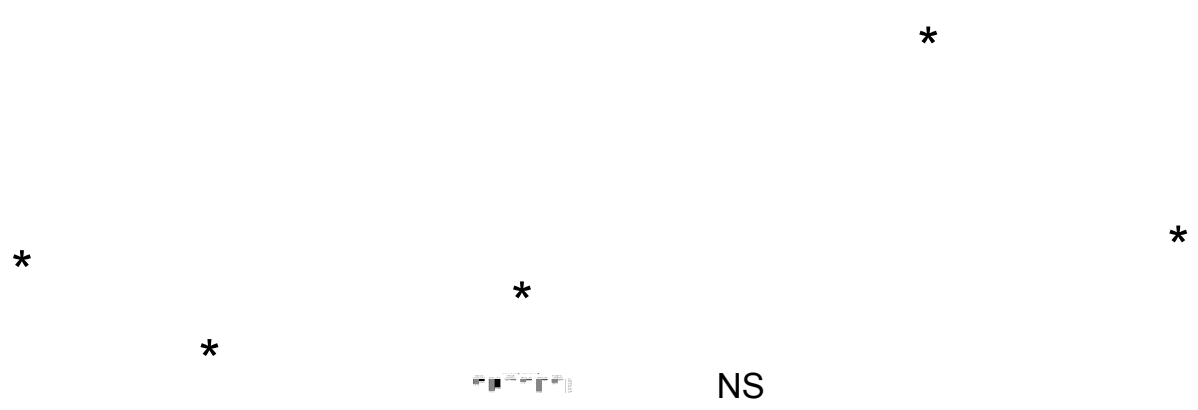

Gráfico 3: Distribuição da síndrome metabólica e de seus componentes, conforme o perfil do IMC

Seguindo as análises pelo perfil do IMC, os dados mostraram que a síndrome metabólica apresentou-se com mais frequência nas crianças e adolescentes com obesidade $(41,2 \%)$ e sobrepeso $(30,8 \%)$ do que nas eutróficas $(1,8 \%)$, com diferença estatisticamente significativa $\left(\chi^{2}=37,190 ; p=0,000\right)$.

Finalmente, analisou-se a frequência dos fatores de risco cardiovasculares nas crianças e adolescentes com síndrome metabólica. Os componentes mais observados dentre aqueles com síndrome metabólica foram o HDL-colesterol baixo $(100 \%)$, a obesidade abdominal $(92 \%)$ e a hipertrigliceridemia (76\%). Comparando-se os meninos e as meninas com síndrome metabólica, a frequência dos componentes foi semelhante entre os gêneros $(p>0,05)$ (GRÁFICO 4). 
Gráfico 4: Distribuição dos fatores de risco cardiovasculares, conforme o gênero, nos es colares com síndrome metabólica

\section{Discussão}

Os fatores de risco cardiovascular e complicações metabólicas têm aumentado na população pediátrica. Nesta pesquisa, a freqüência de três ou mais fatores, ou seja, de SM, foi de 13,7\% no total dos escolares e não apresentou diferenças significativas entre os gêneros. Tal proporção foi relativamente alta, tendo em vista que representa a amostra como um todo, independente do perfil do IMC.

Cook et $a l .{ }^{(7)}$ avaliaram a síndrome metabólica em adolescentes americanos e encontraram 4,2\% de prevalência na população estudada, enquanto que Agirbasli et al. ${ }^{(19)}$ observaram a presença de síndrome metabólica em 2,2\% em crianças e adolescentes turcos. Frequências de síndrome metabólica semelhante ao deste estudo foi verificado por pesquisadores que avaliaram somente crianças e adolescentes com sobrepeso e obesidade, como o realizado por Ferreira, Oliveira e França $^{(9)}$, em que $17,3 \%$ das crianças obesas apresentaram síndrome metabólica. As diferenças nas frequências dos estudos podem estar relacionadas à época de coleta de dados, refletindo modificações temporais de maior morbidade e aumento do excesso de peso na população infanto-juvenil a cada ano. $\mathrm{O}$ alto índice de síndrome metabólica neste estudo pode ser explicado pela elevada proporção de escolares com excesso de peso corporal.

Além disso, as diferenças entre os resultados das pesquisas podem ser atribuídas aos aspectos metodológicos ${ }^{(20)}$. A utilização de um critério não garante total comparabilidade entre os estudos, uma vez que os pontos de corte para os componentes da síndrome metabólica podem variar. Chen e Berenson ${ }^{(21)}$ ressaltam a importância de uma definição universal e colocam que há limitação dos critérios para classificar a síndrome metabólica em populações pediátricas. 
Neste estudo, na avaliação de freqüência de SM foi diagnosticada em 1,8\% das crianças eutróficas pela classificação do IMC, corroborando com outros estu$\operatorname{dos}^{(7)}$. Estes achados são fatores preocupantes revelando que outros fatores além do excesso de gordura corporal estão contribuindo para a síndrome metabólica. Ao considerar os indivíduos com excesso de peso, a freqüência aumentou, semelhante ao estudo de Buff et al. ${ }^{(10)}$ que apresentou uma amostra de $42,9 \%$ de SM em obesos.

Dentre os critérios diagnósticos para a síndrome metabólica, o HDL-c baixo é o componente de maior alteração, atingindo $63,2 \%$ do total das crianças e adolescentes. Corroborando com este estudo, Castillo et al. ${ }^{(22)}$ observaram valores de $85,4 \%$ de HDL-c baixo.Quando analisado somente os escolares com síndrome metabólica, foi observado um percentual de $100 \%$ de HDL-c baixo.

Analisando o grupo com excesso de peso, observa-se que 76,8\% da amostra possui HDL-c baixo. No estudo de Leite et al. ${ }^{(18)}$ foram encontrados valores de HDL-c alterado em $21 \%$ nas meninas e $31 \%$ nos meninos com peso adequado. Este valor aumentou para $63 \%$ e $77 \%$ respectivamente, quando analisado o grupo com obesidade. A redução do HDL-c pode estar relacionada ao maior catabolismo devido à maior concentração de triglicérides nestas partículas ${ }^{(4)}$. De certa forma a diminuição do HDL-c pode estar relacionado ao sedentarismo, pois a atividade física é um fator relevante na elevação do HDL-c ${ }^{(23)}$ e uma dificuldade no transporte reverso.

A literatura ainda é controversa se a dislipidemia pode ser evento primário e ou secundário a obesidade infantil ${ }^{(24)}$. Gama, Carvalho e Miranda ${ }^{(25)}$, no Rio de Janeiro, observaram $10,7 \%$ das crianças apresentavam sobrepeso e em $68,4 \%$ foi observado algum tipo de alteração no perfil lipídico.

Outro componente da síndrome metabólica com alto valor de alteração foi à obesidade abdominal, sendo observada em $33,4 \%$ da amostra, sendo $32,4 \% \mathrm{em}$ meninos e $34,2 \%$ em meninas. Ao considerar somente indivíduos com excesso de peso, a freqüência foi para $79,7 \%$. Resultados semelhantes foram encontrados em outro estudo, realizado em Santo André (SP), com 59 crianças com sobrepeso e obesidade, verificaram-se resultados acima dos achados, com $88,1 \%$ das crianças apresentando obesidade abdominal ${ }^{(10)}$. Este valor aumenta quando analisado somente os escolares com síndrome metabólica (92\%).

A obesidade central tem sido apontada como um dos principais fatores na fisiopatogênese da síndrome metabólica ${ }^{(26)}$, pois é fator determinante para o desenvolvimento de concentrações adversas de dislipidemia e resistência à insulina em populações pediátricas ${ }^{(27)}$. Além disso, é apontada como fator de risco independente para o desenvolvimento de doenças cardiovasculares, sendo classificada como o componente de maior valor preditivo para a síndrome metabólica ${ }^{(4)}$. Segundo Guimarães e Guimarães ${ }^{(28)}$ esta medida elevada é responsável pelas maiores alterações na resistência à insulina, que por sua vez afeta o metabolismo lipídico e na pressão arterial. 
Em estudo longitudinal de 10 anos, Morrison et al. ${ }^{(29)}$ acompanharam os fatores de risco para o desenvolvimento da síndrome metabólica em meninas observaram que para cada aumento de $1 \mathrm{~cm}$ na circunferência da cintura, o risco de desenvolver SM aumentava 7,4\%. Este fato reforça a importância de incluir a medida da circunferência abdominal em rotinas de avaliação antropométrica na escola. Desta forma, amplia-se a abordagem preventiva e de diagnostico precoce identificando escolares com maiores risco metabólicos, que contribuem para o desenvolvimento de doenças cardiovasculares.

De acordo com os resultados obtidos, conclui-se que a frequência de síndrome metabólica em escolares esta alta, e dentre os seus componentes, o HDL-c baixo e a obesidade abdominal são os mais frequentes. Os resultados corroboram com as atuais recomendações para a prevenção da obesidade e o incentivo à prática de atividade física regular, principalmente na redução da obesidade visceral e aumento das concentrações do HDL-c.

\title{
FREQUENCY OF METABOLIC SYNDROME IN SCHOLARS
}

\begin{abstract}
Objective: To assess the frequency of metabolic syndrome (MS) in children and adolescent with and without overweight from school of Curitiba. Methods: Participated 182 subjects aged between $10-16$ years. They were classified as having MS since they present 3 or more Adult Treatment Panel III (ATP III) criteria, adapted by Leite et al (2009). The T test and the Chi-square test was used $(\mathrm{p}<0,05)$. Results: MS was present in $13.7 \%$, with similar proportions of boys and girls $\left(\chi^{2}=0,12 ; p=0,913\right)$. MS was more frequently observed in scholars with overweight compared to normal weight $\left(\chi^{2}=36,019\right.$; $\mathrm{p}=0,00)$. The components most affected were: HDL-C and waist circumference. Conclusion: The results indicated a high MS frequency in children and adolescents with overweight.
\end{abstract}

Keywords: Child. Adolescent. Obesity. Abdominal Circumference. Insulin Resistance.

\section{FRECUÉNCIA DEL SÍNDROME METABÓLICO EN ESTUDIANTES}

\section{Resumen}

Objetivo: Comparar la frecuencia del síndrome metabólico (SM) en los niños y adoles centes con y sin exceso de peso, de la escuela de Curitiba. Métodos: Participaron 182 sujeto, entre 10 y 16 años de edad. El SM fue clasificado por la presencia de 3 o más de los criterios del Adult Treatment Panel III (ATPIII), adaptado por Leite et al (2009). Se utilizó el test $\mathrm{T}$ Student y test chi-cuadrado $(\mathrm{p}<0,05)$. Resultados: El SM ocurrió en $13,7 \%$ con proporciones semejantes entre géneros $\left(\chi^{2}=0,12 ; p=0,913\right)$. El SM se vio en frecuencia en los estudiantes con exceso de peso en relación con adecuado $\left(\chi^{2}=36,019 ; p=0,000\right)$. 
Los componentes más alterados son: HDL-c y circunferencia de la cintura. Conclusión: Se vio la elevación de la proporción del SM en niños y adolescentes con exceso de peso.

Palabras-clave: Niño. Adolescente. Obesidad. Circunferencia Abdominal. Resistencia a la Insulina.

\section{Referências}

BRANDÃO, A. P. et al. I Diretriz Brasileira de Diagnóstico e Tratamento da Síndrome Metabólica. Arquivos Brasileiros de Cardiologia, São Paulo, v. 84, p.118, abr., 2005. Suplemento 1.

MCLELLAN, K. C. P. et al. Diabetes mellitus do tipo 2, síndrome metabólica e modificação do estilo de vida. Revista de Nutrição, Campians, v. 20, n. 5, p. 515524, set./out., 2007.

MANNA, T. D.; DAMIANI, D.; SETIAN, N. Síndrome metabólica: revisão. Pediatria, São Paulo, v. 28, n. 4, p. 272-277, 2006.

DESPRES, J. P. et al. Distribution on et metabolism des masses grasses. Diabetes Metabolism, p. 209-214, 2001.

VOLP, A. C. et al. Capacidade dos biomarcadores inflamatórios em predizer a síndrome metabólica. Arquivos Brasileiros de Endocrinologia e Metabolismo, São Paulo, v. 52, n. 3, p.537-549, abr., 2008.

COOK, S. et al. Prevalence of a metabolic syndrome phenotype in adolescents. Archives of Pediatrics and Adolescent Medicine, Chicago, v. 157, n. 8, p.821827, ago., 2003.

FERRANTI, S. D. et al. Prevalence of the metabolic syndrome in American adolescents. Circulation, Hagerstown, v. 110, n. 16, p. 2494-2497, out., 2004.

FERREIRA, A. P.; OLIVEIRA, C. E. R.; FRANÇA, N. M. Síndrome metabólica em crianças obesas e fatores de risco para doenças cardiovasculares de acordo com a resistência à insulina (HOMA-IR). Jornal de Pediatria, Rio de Janeiro/Porto Alegre, v. 83, n. 1, p.21-26, jan./fev., 2007.

BUFF, C. G. et al. Freqüência de Síndrome Metabólica em crianças e adolescentes com sobrepeso e obesidade. Revista Paulista de Pediatria, São Paulo, v. 25, n. 3, p. 221-226, set., 2007. 
MOSER, D. C. Indicadores antropométricos e pressão arterial em escolares da rede municipal de Curitiba-PR. 2010. 162 f. Dissertação (Mestrado em Educação Física)-Departamento de Educação Física, Universidade Federal do Paraná, Curitiba, 2010.

LAKKA, H. M.. The metabolic syndrome and total and cardiovascular disease mortal in middle-aged men. JAMA, v. 288, n. 21, p. 2709-16, dez., 2002.

LOHMAN, T. G.; ROCHE, A. F.; MARTORELL, R. Anthropometric standardization reference manual. Champaign: Human Kinetics; 1988.

KUCZMARSKI, R. J. et al. CDC growth charts: United States. Advance data from vital and health statistics. Maryland: National Center for Health Statistics, 2000. p. 1-28.

FERNANDEZ, J. R. et al. Waist circumference percentiles in nationally representative samples of African-American, European-American, and Mexican-American children and adolescents. Journal of Pediatrics, v. 145, n. 4, p. 439-444, out., 2004.

SOCIEDADE BRASILEIRA DE CARDIOLOGIA; SOCIEDADE BRASILEIRA DE HIPERTENSÃO; SOCIEDADE BRASILEIRA DE NEFROLOGIA. VI Diretrizes Brasileiras de Hipertensão. Arquivo Brasileiro de Cardiologia, São Paulo, v. 95 , n. 1, p. 1-51, 2010. Suplemento 1.

TANNER, J. M. Normal growth and techniques of growth assessment. Journal of Clinical Endocrinology \& Metabolism, v. 15, n. 3, p. 411-451, 1986.

LEITE, N. et al. Effects of physical exercise and nutritional guidance on metabolic syndrome in obese adolescents. Revista Brasileira de Fisioterapia, São Carlos, v.13, n. 1, p. 73-81, jan./fev., 2009.

AGIRBASLI, M. et al. Metabolic syndrome in Turkish children and adolescents. Metabolism, v. 55, n. 8, p. 1002-06, ago., 2006.

MORAES, A. C. F. et al. Prevalência de síndrome metabólica em adolescentes: uma revisão sistemática. Caderno de Saúde Pública, Rio de Janeiro, v. 25, n. 6, p. 1195-1202, jun., 2009.

CHEN, W.; BERENSON, G. S. Síndrome metabólica: definição e prevalência em crianças. Jornal de Pediatria, Rio de Janeiro/Porto Alegre, v. 83, n. 1, p.1-3, jan./fev., 2007. 
CASTILLO, E. H. et al. Body mass index and the prevalence of metabolic syndrome among children and adolescents in two Mexican populations. Journal of Adolescent Health, v. 40, n. 6, p.521-526, jun., 2007.

PARENTE, E. B. et al. Perfil lipídico em crianças obesas: Efeitos de dieta hipocalórica e atividade física aeróbica. Arquivos Brasileiros de Endocrinologia e Metabolismo, São Paulo, v. 50, n. 3, p. 499-504, jun., 2006.

GIULIANO, I. C. B.; CARAMELI, B. Dislipidemia na infância e adolescência. Pediatria, São Paulo, v. 29, n. 4, p. 275-85, 2008.

GAMA, S. R.; CARVALHO, M. S.; MIRANDA, C. R. M. Prevalência em crianças de fatores de risco para as doenças cardiovasculares. Caderno de Saúde Pública, Rio de Janeiro, v. 23, n. 9, p. 231-36, set., 2007.

LOPES, H. F. Aspectos históricos da síndrome metabólica. Síndrome metabólica: uma abordagem multidisciplinar. São Paulo: Atheneu; 2007

FREEDMAN, D. S. et al. Relation of circumferences and skinfold tchicknesses to lipid and insulin concentrations in children and adolescents: the Bogalusa Heart Study. American Journal of Clinical Nutrition, p. 308-17, 1999.

GUIMARÃES, I. C. B.; GUIMARÃES, A. C. Síndrome metabólica na infância e adolescência. Um fator maior de risco cardiovascular. Revista Baiana de Saúde Pública, v.30, p. 349-62, 2006.

MORRISON, J. A. et al. Development of the metabolic syndrome, in black and white adolescent girls: a longitudinal assessment. Pediatrics, v. 116, n. 5, p. 117882, nov., 2005.

Recebido em: 14/08/2012

Revisado em: 07/04/2013

Aprovado em: 04/11/2013

Endereço para correspondência:

annakapp@hotmail.com

Ana Cláudia Kapp Titski

Universidade Federal do Paraná

Rua XV de Novembro, 1299

Centro, Curitiba - PR,

CEP: 80060-000 